\title{
Effectiveness of varenicline versus nicotine replacement therapy for smoking cessation with minimal professional support: evidence from an English population study
}

Manuscript revised according to reviewer comments from Psychopharmacology on Revision 1

Daniel Kotz $\mathrm{PhD}^{1,2^{*}}$, Jamie Brown PhD ${ }^{1}$, Robert West $\mathrm{PhD}^{1}$

${ }^{1}$ Department of General Practice, CAPHRI School for Public Health and Primary Care, Maastricht University Medical Centre, Maastricht, the Netherlands. ${ }^{2}$ Cancer Research UK Health Behaviour Research Centre, University College London, WC1E 6BT, UK

Number of words: 2,935 (main text); 254 (abstract)

Tables: 2; Figures: 0

Key words: smoking cessation, varenicline, nicotine replacement therapy on prescription, crosssectional population survey

*Correspondence to: Dr. Daniel Kotz, CAPHRI School for Public Health and Care, Maastricht University Medical Centre, P.O. Box 616, 6200 MD Maastricht, Maastricht, the Netherlands. Telephone: +3143 38 82893. FAX: +31 4336 19344. E-mail: d.kotz@maastrichtuniversity.nl. Website: www.daniel-kotz.de.

Statement of competing interests: RW has undertaken research and consultancy and received travel funds from companies that develop and manufactures smoking cessation medications. He has a share of a patent for a novel nicotine delivery device. He is a trustee of the stop-smoking charity, QUIT and Co-direct of the National Centre for Smoking Cessation and Training. DK has received an unrestricted research grant from Pfizer for a smoking cessation trial. JB has received an unrestricted research grant from Pfizer. This study is partly funded by Pfizer under an investigator initiated award. 


\section{ABSTRACT}

\section{Rationale}

Limited evidence from randomised controlled trials suggests that varenicline might be more effective than nicotine replacement therapy (NRT) in achieving abstinence from smoking. The comparative effectiveness of varenicline when prescribed under routine circumstances and in the general population has not been tested.

\section{Objectives}

To compare the abstinence rates of smokers trying to stop having used varenicline versus NRT on prescription $(\mathrm{Rx})$ when provided with minimal professional support in the general population while adjusting for key potential confounders.

\section{Methods}

A large survey of a representative sample of the English population. Participants were 1,579 adults who smoked within the previous 12 months and made at least one quit attempt with varenicline or NRT Rx in their most recent quit attempt. The main outcome measure was self-reported abstinence up to the time of the survey, adjusted for key potential confounders including urges to smoke. A sensitivity analysis was conducted in subsamples in which the quit attempt started up to 6 months or more than 6 months ago.

\section{Results}

The adjusted odds of abstinence in users of varenicline were $1.76(95 \% \mathrm{Cl}=1.22-2.53)$ times higher compared with users of NRT Rx. However, there was no detectable difference in the subsample of smokers who started their quit attempt more than 6 months ago (adjusted OR=1.03, 95\% $\mathrm{Cl}=0.54$ 1.96).

\section{Conclusions}

Varenicline use with minimal professional support in the general population of smokers appears more effective than NRT Rx in achieving short-term abstinence. However, this effect may disappear in the long term. Research is needed to confirm this and establish what may underlie it. 


\section{INTRODUCTION}

The World Health Organization (WHO) estimates that smoking kills nearly six million people each year and that up to half the users who do not stop will die of a tobacco-related disease.(World Health Organisation (WHO), 2011) Every year that someone continues to smoke after early middle age loses them 3 months of life expectancy.(Doll et al., 2004) It is therefore important that every quit attempt has the best possible chance of success.

Several pharmacological treatments can improve the chance of successful quitting. Varenicline, a partial $\alpha 4 \beta 2$ receptor agonist, has been shown to be more effective than placebo and bupropion in achieving long-term abstinence from smoking.(Gonzales et al., 2006; Jorenby et al., 2006) Few randomised controlled trials, however, have directly compared the efficacy of varenicline with nicotine replacement therapy (NRT), which has been the most widely used smoking cessation drug for decades. One large-scale $(\mathrm{N}=746)$ multi-national trial showed that, in the context of intensive behavioural support, varenicline was more effective than NRT transdermal patch in achieving short-term (end-of-treatment) abstinence.(Aubin et al., 2008) However, this trial as well as a medium-scale ( $\mathrm{N}=272$ ) trial from Iran (Heydari et al., 2012) and a small-scale $(\mathrm{N}=28)$ trial from Japan (Tsukahara et al., 2010) did not provide strong evidence for the long-term superiority (abstinence at 6- to 12-months post-treatment).

Randomised controlled trials, even what are termed "effectiveness trials" that seek to simulate as far as possible the routine clinical environment, need to be supplemented by "real world" studies in order to establish generalisability. This is because requiring informed consent to randomisation is potentially an important threat to external validity. Such real world studies are not a substitute for randomised trials, but complement them. They necessarily use epidemiological methods and rely on correlational evidence with statistical adjustment for potential confounding variables. Several cohort studies comparing the "real world" effectiveness of varenicline and NRT during routine treatment in clinical settings have produced mixed results.(Biazzo et al., 2010; Brose et al., 2013; Brose et al., 2011; Dhelaria et al., 2012; Sicras Mainar et al., 2011; Stapleton et al., 2008; Steinberg et al., 2011) These studies often include smokers attending specialised stop-smoking services which limit the generalisability of findings because in the most common scenario medication is prescribed with only minimal professional support involving brief instructions on use. Furthermore, observational studies must take account of the fact that smokers who selfselect to use a certain medication may differ with regard to factors that have an effect on 
treatment outcome, in particular their level of cigarette dependence.(Borland et al., 2012; Kotz et al., 2011) Another important factor is that use of pharmacological treatments to aid smoking cessation may be different outside of clinical trials. Users may underdose and in the case of NRT they may use it singly or with more than one form of NRT at the same time or consecutively. There is evidence that combination NRT (e.g., using transdermal patch plus a faster acting form such as nicotine gum) is more effective than single form.(Fiore et al., 2008; Stead et al., 2008)

The current study is the first to compare the effectiveness of varenicline with NRT on prescription (NRT Rx) when provided with minimal professional support in the general population and while adjusting for key potential confounding factors.

\section{METHODS}

We used data from the "Smoking Toolkit Study", which is an ongoing research programme designed to provide information about smoking cessation and factors that promote or inhibit it at a population level.(Fidler et al., 2011a) Each month a new sample of approximately 1,800 adults in England aged 16 and over completes a face-to-face computer-assisted survey, of whom approximately 450 are smokers. The methods have been described in full elsewhere and have been shown to result in figures for key variables such as smoking prevalence that are nationally representative.(Fidler, et al., 2011a)(www.smokinginengland.info)

TNS-BMRB collects the data as part of their monthly omnibus surveys on behalf of researchers at University College London. The surveys use a form of random location sampling. England is split into 165,665 Output Areas, each comprising approximately 300 households. These Output Areas are stratified by ACORN characteristics (an established geo-demographic analysis of the population; http://www.caci.co.uk/acorn) and then randomly selected to be included in the lists of the interviewers. Interviewers travel to the selected areas and perform interviews with one participant per household until quotas based upon factors influencing the probability of being at home (working status, age, and gender) are fulfilled. Morning interviews are avoided to maximise participant availability. 


\section{Study population}

For the current study, we used aggregated data from respondents to the survey in the period from November 2006 (the start of the survey) to September 2012 (the latest wave of the survey for which data were available), who smoked either cigarettes (including hand-rolled) or any other tobacco product (e.g., pipe or cigar) daily or occasionally at the time of the survey or during the preceding 12 months. We included those who made at least one quit attempt in the preceding 12 months, assessed by asking: "How many serious attempts to stop smoking have you made in the last 12 months? By serious attempt I mean you decided that you would try to make sure you never smoked again. Please include any attempt that you are currently making and please include any successful attempt made within the last year." We also asked how long ago the most recent quit attempt started and categorised respondents into those who started their quit attempt in the last week or up to 6 months ago and those who started their quit attempt more than 6 months ago.

To identify methods used to stop smoking, respondents were asked "Which, if any, of the following did you try to help you stop smoking during the most recent serious quit attempt?" Respondents could select any of the following: "nicotine replacement product on prescription or given to you by a health professional, Champix (varenicline), attended a stop smoking group, attended one or more stop smoking one-to-one counselling\advice \support session\s, nicotine replacement product (e.g., patches\gum\inhaler) without a prescription."

We included respondents who used either varenicline or NRT on prescription (Rx) during their most recent quit attempt and excluded those who used these medications in combination with a stop smoking group or counselling (9.9\% of medication users) and a nicotine replacement product obtained without prescription. This resulted in a sample of smokers who tried to quit with varenicline or NRT Rx which was assumed to be combined with minimal professional support.

\section{Measurements}

Our primary outcome was self-reported non-smoking up to the time of the survey. Respondents were asked: "How long did your most recent serious quit attempt last before you went back to smoking?". Those responding "I am still not smoking" were defined as non-smokers. Previous research has shown that self-reported abstinence in surveys of this kind closely reflects true smoking rates and is not subject to the kind of biases observed in clinical trials where there is social pressure to claim abstinence.(West et al., 2007; Wong et al., 2012) 
We measured a range of variables that are potentially associated with the use of smoking cessation treatments and may also have an effect on the outcome. First, time spent with urges to smoke was assessed by asking: "How much of the time have you felt the urge to smoke in the past 24 hours? Not at all (coded 1), a little of the time (2), some of the time (3), a lot of the time (4), almost all of the time (5), all of the time (6)". Second, strength of urges to smoke was measured by asking "In general, how strong have the urges to smoke been?": slight (1), moderate (2), strong (3), very strong (4), extremely strong (5). This question was coded "0" for smokers who responded "not at all" to the previous question. Different measures of dependence exist but urges to smoke have been found to be a better predictor of relapse than the more common Fagerström Test for Nicotine Dependence and its components in this particular population.(Fidler et al., 2011b) Demographic characteristics we took into account were age, sex, and social grade $(A B=$ managerial and professional occupations, $\mathrm{C} 1=$ intermediate occupations, $\mathrm{C} 2=$ small employers and own account workers, $\mathrm{D}=$ lower supervisory and technical occupations, and $\mathrm{E}=$ semi-routine and routine occupations, never workers, and long-term unemployed). Furthermore, we measured the number of quit attempts in the last year prior to the one in question, time since the quit attempt in question was initiated, and whether smokers had tried to quit abruptly or gradually.

\section{Data analysis}

The simple associations between potential confounders and use of varenicline vs. NRT Rx were assessed with ANOVA for continuous variables and Pearson's $\chi^{2}$ for categorical variables.

For the primary analysis, we used a multiple logistic regression model in which we regressed the outcome measure (non-smoker vs. smoker) on the effect measure (varenicline vs. NRT Rx), adjusted for the above mentioned potential confounders and wave of the survey. The model included interaction terms between time since last quit attempt and time spent with urges, and between time since last quit attempt and strength of urges to smoke. These two latter interaction terms were used to account for the fact that urges to smoke following the quit attempt will be influenced by whether the respondent is currently abstinent and the duration of abstinence.

In a sensitivity analysis, we assessed potential differential bias in recall of quit attempts by testing the association between time since the last quit attempt started and type of medication used during that quit attempt (varenicline or NRT Rx) with Pearson's $\chi^{2}$. Subsequently, we ran the model 
of the primary analysis separately for the sub-samples of smokers who started their last quit attempt less versus more than 6 months ago to assess any difference in short- versus long-term effectiveness of the two medications.

All analyses were performed with complete cases. Respondents with missing data on one or more of the variables were excluded ( $5 \%$ of the initial sample).

\section{RESULTS}

The study population consisted of 1,579 respondents; 1,329 (84.2\%) who smoked and $250(15.8 \%)$ who were abstinent at the time of the survey. A total of $540(34.2 \%)$ respondents had used varenicline and 1,039 (65.8\%) had used NRT Rx during their most recent quit attempt.

Associations between characteristics of the sample and use of varenicline or NRT Rx are presented in Table 1. There was no difference in age and sex but a non-linear association with the categories of social grade. NRT Rx users were more likely to have made more quit attempts in the past and to have stopped abruptly without cutting down first during their most recent quit attempt. They also reported higher levels of urges to smoke. There was no difference in time since the last quit attempt started.

The results of our primary analysis are presented in Table 2 . The adjusted odds of non-smoking in users of varenicline were $1.76(95 \% \mathrm{Cl}=1.22-2.53)$ times higher compared with users of NRT Rx. Although there was no statistically significant interaction between time since last quit attempt and type of medication used during that attempt (adjusted $\mathrm{OR}=0.54,95 \% \mathrm{Cl}=0.26-1.12$ ), the table shows that the adjusted odds of non-smoking in users of varenicline were not different from users of NRT Rx in smokers who started their last quit attempt more than 6 months ago (adjusted $\mathrm{OR}=1.03,95 \% \mathrm{Cl}=0.54-1.96)$.

\section{DISCUSSION}

In a national survey of the English population, use of varenicline during a quit attempt was associated with a 1.76 times higher rate of success compared with NRT Rx. However, in the subsample of smokers who started their quit attempt more than 6 months ago, varenicline did not appear to be associated with a higher rate of success. 
The overall odds ratio in this study was slightly higher than those from the two published randomised controlled trials comparing varenicline with single form NRT (Aubin, et al., 2008; Heydari, et al., 2012) and larger than one small trial (Tsukahara, et al., 2010). It was also similar to the odds ratios for varenicline versus single form NRT from observational studies in which intensive behavioural support was provided (Brose, et al., 2011; Stapleton, et al., 2008). However, the overall odds ratio in this study was greater than has been found when varenicline has been compared in observational studies with combination NRT (Brose, et al., 2013; Stapleton, et al., 2008).

Although the interaction between time since last quit attempt and type of medication used was not statistically significant, our sensitivity analysis showed a significantly higher odds of successful quitting in varenicline users in the subsample who started their quit attempt less than 6 months ago $(\mathrm{OR}=2.20(95 \% \mathrm{Cl}=1.39-3.47))$. In the subsample who started their quit attempt more than 6 months ago, however, varenicline appeared to be no better than NRT Rx (OR=1.03 (0.54-1.96)). There was no detectable association between time since the last quit attempt started and type of medication used, so it is unlikely that the latter finding is due to differential recall bias (i.e., that failed quit attempts that started more than 6 months ago are more likely to be forgotten when they were made with NRT Rx than with varenicline). If these results are unbiased, they would indicate that the increased effectiveness of varenicline compared with NRT Rx seen in the shortterm is not sustained in the long-term in smokers from the general population who used these medications without specialist behavioural support. This finding could be due to lower adherence to NRT Rx than to varenicline during the first months following the quit date, or by longer term use of NRT Rx (e.g., longer than three months; the usual duration of treatment with varenicline). However, it should be noted that the power to detect both the interaction and the effect in the subsample was low.

The main limitations of this study relate to the cross-sectional survey design. The most important potential bias in our study is confounding by indication (the fact that smokers who self-select to use varenicline or NRT Rx may differ with regard to factors that have an effect on abstinence from smoking). We reduced the risk of confounding by adjusting for urges to smoke, age, sex, social grade, abrupt quitting vs. cutting down, and previous quit attempts. However, we were not able to measure dependence at the time of treatment initiation. We adjusted for urges to smoke at the time of the survey as this measure has been shown to predict relapse best in this population and 
because we were unable to adjust for other existing measures of dependence. This approach could introduce bias when comparing methods of quitting that might differentially affect urges. However, if such bias occurred the results of an analysis adjusting for urges to smoke would underestimate the benefit of varenicline because varenicline appears to reduce urges to smoke during quitting more than NRT.(Aubin, et al., 2008) Thus, our finding that varenicline was associated with greater success rates cannot be explained by this.

We adjusted for several potential confounders, but not all factors associated with self-selection of treatment were measured in our survey, such as previous use of NRT or varenicline during a quit attempt, physical health, mental health (Taggar et al., 2012) or psychological distress (Lawrence et al., 2011). The fact that those using NRT Rx had higher urges to smoke, which would be expected to be associated with these variables, suggests that this group may have been harder to treat. However, another explanation for the difference in urges to smoke between varenicline and NRT Rx users we observed may be that varenicline had a larger effect on reducing urges to smoke. Motivation to quit may also be positively associated with both use of treatment and success. However, population studies have generally not found an association between motivation to quit and success of quit attempts.(Vangeli et al., 2011) The difference in abrupt quitting vs. cutting down could possibly be due to spontaneous reduction of smoking during the up-titration phase in the indicated use of varenicline.

Our survey did not contain data on the amount of professional support received by the individual smoker apart from smokers not endorsing the response option indicating that they had attended counselling/advice/support sessions. The issuing of a prescription would normally require personal contact with a physician which sets a lower limit on the amount of professional support received but it is possible that participants received support below a level that they would have regarded as counselling. Furthermore, we did not have information on what proportion of the respondents used single form or combination NRT.

Regarding our outcome variable, it was not feasible in our large population study to biochemically validate self-reported abstinence. This would be a serious limitation in randomized controlled trials because of the possibility of differential likelihood of falsely claiming abstinence by participants in the active treatment.(West et al., 2005) However, in population surveys the misreporting rate is low.(West, et al., 2007; Wong, et al., 2012) A major strength of our study is 
the use of a large, representative sample of the English population. Our study included all smokers aged 16 years or older who made a quit attempt in the 12 months preceding the survey, including those who smoke less than 10 cigarettes per day. This subgroup constitutes one third of current smokers in England (Kotz et al., 2012) and is usually excluded from clinical trials.

In conclusion, this study provided support for a short-term benefit for varenicline compared with NRT as used by the general population of smokers with limited behavioural support. However, we cannot be confident that this advantage is maintained long term. This is an important area for future research. 


\section{ACKNOWLEDGEMENTS}

The Smoking Toolkit Study is funded by the English Department of Health, Cancer Research UK, Pfizer, GlaxoSmithKline, and Johnson and Johnson. Pfizer, Johnson and Johnson, and GlaxoSmithKline are manufacturers of smoking cessation products who had no involvement in the design of the study, collection, analysis or interpretation of the data, the writing of the report, or the decision to submit the paper for publication. 


\section{REFERENCES}

Aubin, H. J., Bobak, A., Britton, J. R., Oncken, C., Billing, C. B., Jr., Gong, J., . . Reeves, K. R. (2008). Varenicline versus transdermal nicotine patch for smoking cessation: results from a randomised open-label trial. Thorax, 63(8), 717-724. doi: 10.1136/thx.2007.090647

Biazzo, L. L., Froshaug, D. B., Harwell, T. S., Beck, H. N., Haugland, C., Campbell, S. L., \& Helgerson, S. D. (2010). Characteristics and abstinence outcomes among tobacco quitline enrollees using varenicline or nicotine replacement therapy. Nicotine \& Tobacco Research, 12(6), 567-573. doi: 10.1093/ntr/ntq045

Borland, R., Partos, T. R., \& Cummings, K. M. (2012). Systematic Biases in Cross-sectional Community Studies may Underestimate the Effectiveness of Stop-Smoking Medications. Nicotine \& Tobacco Research, 14(12), 1483-1487. doi: 10.1093/ntr/nts002

Brose, L., West, R., \& Stapleton, J. (2013). Comparison of the effectiveness of varenicline and combination nicotine replacement therapy for smoking cessation in clinical practice. Mayo Clin Proc, 88(3), 226-233.

Brose, L. S., West, R., McDermott, M. S., Fidler, J. A., Croghan, E., \& McEwen, A. (2011). What makes for an effective stop-smoking service? Thorax. doi: 10.1136/thoraxjnl-2011-200251

Dhelaria, R. K., Friderici, J., Wu, K., Gupta, E., Khan, C., \& Rothberg, M. B. (2012). Effectiveness of varenicline for smoking cessation at 2 urban academic health centers. European Journal of Internal Medicine, 23(5), 461-464.

Doll, R., Peto, R., Boreham, J., \& Sutherland, I. (2004). Mortality in relation to smoking: 50 years' observations on male British doctors. BMJ, 328(7455), 1519-1510.

Fidler, J., Shahab, L., West, O., Jarvis, M., McEwen, A., Stapleton, J., . . West, R. (2011a). 'The Smoking Toolkit Study': A national study of smoking and smoking cessation in England. BMC Public Health, 11(1), 479.

Fidler, J. A., Shahab, L., \& West, R. (2011b). Strength of urges to smoke as a measure of severity of cigarette dependence: comparison with the Fagerstrom Test for Nicotine Dependence and its components. Addiction, 106(3), 631-638. doi: 10.1111/j.1360-0443.2010.03226.x

Fiore, M. C., Jaén, C. R., Baker, T. B., Bailey, W. C., Benowitz, N. L., Curry, S. J., . . . et al. (2008). Treating tobacco use and dependence: 2008 update. Rockville, MD: US Department of Health and Human Services.

Gonzales, D., Rennard, S. I., Nides, M., Oncken, C., Azoulay, S., Billing, C. B., . . . for the Varenicline Phase 3 Study Group. (2006). Varenicline, an alpha4-beta2 Nicotinic Acetylcholine Receptor 
Partial Agonist, vs Sustained-Release Bupropion and Placebo for Smoking Cessation: A Randomized Controlled Trial. JAMA, 296(1), 47-55.

Heydari, G., Talischi, F., Tafti, S. F., \& Masjedi, M. R. (2012). Quitting smoking with varenicline: parallel, randomised efficacy trial in Iran. [Comparative Study

Randomized Controlled Trial

Research Support, Non-U.S. Gov't]. The international journal of tuberculosis and lung disease : the official journal of the International Union against Tuberculosis and Lung Disease, 16(2), 268-272. doi: 10.5588/ijtld.11.0183

Jorenby, D. E., Hays, J. T., Rigotti, N. A., Azoulay, S., Watsky, E. J., Williams, K. E., . . for the Varenicline Phase 3 Study Group. (2006). Efficacy of Varenicline, an \{alpha\}4beta2 Nicotinic Acetylcholine Receptor Partial Agonist, vs Placebo or Sustained-Release Bupropion for Smoking Cessation: A Randomized Controlled Trial. JAMA, 296(1), 56-63.

Kotz, D., Fidler, J., \& West, R. (2012). Very low rate and light smokers: smoking patterns and cessation-related behaviour in England, 2006-2011. Addiction, 107(5), 995-1002. doi: 10.1111/j.1360-0443.2011.03739.x

Kotz, D., Fidler, J. A., \& West, R. (2011). Did the introduction of varenicline in England substitute for or add to the use of other smoking cessation medications? Nicotine \& Tobacco Research, 13(9), 793-799. doi: doi: 10.1093/ntr/ntr075

Lawrence, D., Mitrou, F., \& Zubrick, S. R. (2011). Non-specific psychological distress, smoking status and smoking cessation: United States National Health Interview Survey 2005. BMC Public Health, 11, 256. doi: 10.1186/1471-2458-11-256

Sicras Mainar, A., Navarro Artieda, R., Diaz Cerezo, S., Marti Sanchez, B., \& Sanz De Burgoa, V. (2011). [Abstinence rates with varenicline compared to bupropion and nicotine replacement therapy for quitting smoking in primary care]. Aten Primaria, 43(9), 482-489. doi: 10.1016/j.aprim.2010.09.010

Stapleton, J. A., Watson, L., Spirling, L. I., Smith, R., Milbrandt, A., Ratcliffe, M., \& Sutherland, G. (2008). Varenicline in the routine treatment of tobacco dependence: a pre-post comparison with nicotine replacement therapy and an evaluation in those with mental illness. Addiction, 103(1), 146-154.

Stead, L. F., Perera, R., Bullen, C., Mant, D., \& Lancaster, T. (2008). Nicotine replacement therapy for smoking cessation. Cochrane Database of Systematic Reviews(1).

Steinberg, M. B., Bover, M. T., Richardson, D. L., Schmelzer, A. C., Williams, J. M., \& Foulds, J. (2011). Abstinence and psychological distress in co-morbid smokers using various 
pharmacotherapies. Drug Alcohol Depend, 114(1), 77-81. doi:

10.1016/j.drugalcdep.2010.06.022

Taggar, J., Coleman, T., Lewis, S., \& Szatkowski, L. (2012). The impact of the Quality and Outcomes Framework (QOF) on the recording of smoking targets in primary care medical records: cross-sectional analyses from The Health Improvement Network (THIN) database. BMC Public Health, 12(1), 329.

Tsukahara, H., Noda, K., \& Saku, K. (2010). A randomized controlled open comparative trial of varenicline vs nicotine patch in adult smokers: efficacy, safety and withdrawal symptoms (the VN-SEESAW study). Circ J, 74(4), 771-778.

Vangeli, E., Stapleton, J., Smit, E. S., Borland, R., \& West, R. (2011). Predictors of attempts to stop smoking and their success in adult general population samples: A systematic review. Addiction, 106(12), 2110-2121. doi: 10.1111/j.1360-0443.2011.03565.x

West, R., Hajek, P., Stead, L., \& Stapleton, J. (2005). Outcome criteria in smoking cessation trials: proposal for a common standard. Addiction, 100(3), 299-303.

West, R., Zatonski, W., Przewozniak, K., \& Jarvis, M. J. (2007). Can we trust national smoking prevalence figures? Discrepancies between biochemically assessed and self-reported smoking rates in three countries. Cancer Epidemiol Biomarkers Prev, 16(4), 820-822.

Wong, S. L., Shields, M., Leatherdale, S., Malaison, E., \& Hammond, D. (2012). Assessment of validity of self-reported smoking status. Health Rep, 23(1), 47-53.

World Health Organisation (WHO). (2011). WHO report on the global tobacco epidemic. Warning about the dangers of tobacco. Geneva. 
Table 1: Associations between sample characteristics and use of varenicline or NRT Rx

\begin{tabular}{|c|c|c|c|}
\hline Variable & $\begin{array}{c}\text { Varenicline } \\
(\mathrm{N}=540)\end{array}$ & $\begin{array}{c}\text { NRT Rx } \\
(\mathrm{N}=1,039) \\
\end{array}$ & $\mathbf{P}$ \\
\hline Age, mean (SD) & $43.3(13.7)$ & $43.4(15.9)$ & 0.92 \\
\hline Female sex & $54.8(296)$ & $59.4(617)$ & 0.81 \\
\hline \multicolumn{4}{|l|}{ Social grade } \\
\hline$A B$ & $8.5(46)$ & $9.1(95)$ & 0.007 \\
\hline $\mathrm{C} 1$ & $24.4(132)$ & $18.5(192)$ & \\
\hline $\mathrm{C} 2$ & $22.0(119)$ & $23.6(245)$ & \\
\hline $\mathrm{D}$ & $21.3(115)$ & $18.3(190)$ & \\
\hline $\mathrm{E}$ & $23.7(128)$ & $30.5(317)$ & \\
\hline \multicolumn{4}{|l|}{ Number of quit attempts in the past year } \\
\hline 1 & 73.9 (399) & $66.2(688)$ & 0.004 \\
\hline 2 & $18.3(99)$ & $20.8(216)$ & \\
\hline 3 & $5.0(27)$ & $7.4(77)$ & \\
\hline 4 or more & $2.8(15)$ & $5.6(58)$ & \\
\hline Time since last quit attempt started & & & 0.24 \\
\hline$<=1$ to 26 weeks & $58.5(316)$ & $61.6(640)$ & \\
\hline 26 to 52 weeks & $41.4(224)$ & 38.4 (399) & \\
\hline Stopped abruptly without cutting down first & $43.2(233)$ & $57.4(596)$ & $<0.001$ \\
\hline Time spent with urges to smoke, mean (SD) & $3.1(1.4)$ & $3.3(1.3)$ & 0.004 \\
\hline Strength of urges to smoke, mean (SD) & $2.0(1.3)$ & $2.3(1.2)$ & $<0.001$ \\
\hline
\end{tabular}

Figures are presented as percentage within varenicline/NRT $\mathrm{Rx}(\mathrm{N})$, unless stated otherwise. Time spent with urges to smoke: 1 (not at all) to 6 (all the time). Strength of urges to smoke: 0 (no urges) to 5 (extremely strong urges). NRT Rx $=$ nicotine replacement therapy on prescription. Social grade: $A B=$ managerial and professional occupations, $C 1=$ intermediate occupations, $\mathrm{C} 2=$ small employers and own account workers, $\mathrm{D}=$ lower supervisory and technical occupations, and $\mathrm{E}=$ semi-routine and routine occupations, never workers, and long-term unemployed. 
Table 2: Adjusted and unadjusted odds ratio of self-reported non-smoking in the full sample and in the two subsamples of respondents who started their quit attempt less/more than 6 months

ago

\begin{tabular}{|c|c|c|}
\hline Smoking cessation treatment & Unadjusted OR (95\%CI) & Adjusted OR (95\% CI) \\
\hline \multicolumn{3}{|l|}{ Full sample $(\mathrm{N}=1,579)$} \\
\hline Varenicline $(\mathrm{N}=540)$ & $1.74(1.32-2.29)$ & $1.76(1.22-2.53)$ \\
\hline NRT Rx (reference) $(\mathrm{N}=1,039)$ & 1 & 1 \\
\hline \multicolumn{3}{|c|}{ Subsample: quit attempt started $<6$ months ( $N=956$ ) } \\
\hline Varenicline $(\mathrm{N}=316)$ & $2.15(1.54-3.00)$ & $2.20(1.39-3.47)$ \\
\hline NRT Rx (reference) $(\mathrm{N}=640)$ & 1 & 1 \\
\hline \multicolumn{3}{|c|}{ Subsample: quit attempt started $>6$ months $(N=623)$} \\
\hline Varenicline $(\mathrm{N}=224)$ & $1.19(0.73-1.94)$ & $1.03(0.54-1.96)$ \\
\hline NRT Rx (reference) $(\mathrm{N}=399)$ & 1 & 1 \\
\hline \multicolumn{3}{|c|}{$\begin{array}{l}\text { Odds ratio (OR) adjusted for age, sex, social grade, number of quit attempts in the last year, time since last quit } \\
\text { attempt started, time spent with urges to smoke, strength of urges to smoke, time since last quit attempt started } * \\
\text { time spent with urges, time since last quit attempt started } * \text { strength of urges to smoke, abrupt versus gradual } \\
\text { quitting, and wave of the survey. } 95 \% \mathrm{Cl}=95 \% \text { confidence interval around OR. NRT Rx = nicotine replacement therapy } \\
\text { on prescription. }\end{array}$} \\
\hline
\end{tabular}

